\title{
Number of Days from Date of Collection to Date of Last Contact
}

National Cancer Institute

\section{Source}

National Cancer Institute. Number of Days from Date of Collection to Date of Last

Contact. NCI Thesaurus. Code C160677.

The number of days from the date of collection of the specimen to the date of last contact. 